\title{
Model for cone directionality reflectometric measurements based on scattering
}

\author{
Susana Marcos, Stephen A. Burns, and Ji Chang He \\ Schepens Eye Research Institute, 20 Staniford Street, Boston, Massachusetts 02114
}

Received November 13, 1997; revised manuscript received March 27, 1998; accepted April 2, 1998

\begin{abstract}
Reflectrometric measurements provide an objective assessment of the directionality of the photoreceptors in the human retina. Measurements are obtained by imaging the distribution at the pupil plane of light reflected off the human fundus in a bleached condition. We propose that scattering as well as waveguides must be included in a model of the intensity distribution at the pupil plane. For scattering, the cone-photoreceptor array is treated as a random rough surface, characterized by the correlation length $T$ (related to the distance between scatterers, i.e., mean cone spacing) and the roughness standard deviation $\sigma$ (assuming random length variations of the cone outer-segment lengths that produce random phase differences). For realistic values of $T$ and $\sigma$ we can use the Kirchhoff approximation for computing the scattering distribution. The scattered component of the distribution can be fitted to a Gaussian function whose width depends only on $T$ and $\lambda$. Actual measurements vary with experimental conditions (exposure time, retinal eccentricity, and $\lambda$ ) in a manner consistent with the scattering model. However, photoreceptor directionality must be included in the model to explain the actual location of the peak of the intensity distribution in the pupil plane and the total angular spread of light. (C) 1998 Optical Society of America [S0740-3232(98)00808-4]
\end{abstract}

OCIS codes: $330.150,330.190,330.200,330.4300,330.5310,330.5370,110.0110,290.0290$.

\section{INTRODUCTION}

In recent years there have been major advances in assessing the human visual system by objectively imaging the light scattered back from the retina. ${ }^{1-9}$ The principal aim of these techniques is to provide objective information on a given aspect of the visual system rapidly and reliably and in a manner that is well tolerated by both normal subjects and patients. Generally, one can get the same information with traditional psychophysical techniques, but the reliability and time saving of the objective techniques make it desirable for eventual clinical purposes. $^{3,4,7,8}$

In this paper we will discuss reflectometric techniques that have been developed to measure photoreceptor directionality. The idea behind these measurements is that when bleached, the photoreceptors will guide light back toward the pupil, just as they guide light arriving from the pupil. The procedure consists of measuring the light returning from the retina is relative to pupil coordinates. Different groups have used slightly different approaches. For example, Van Blockland ${ }^{10}$ measured the scattering diagrams as the exit pupil was scanned along one axis; Gorrand and Delori ${ }^{11}$ scanned both the entry pupil (for illumination) and the exit pupil (for light collection); Burns et $a l .^{6,9}$ imaged the entire distribution of the light returning from the retina through the pupil for any given pupil entry position; and Delint et al. ${ }^{12}$ analyzed digitized images of the fundus reflectance obtained with a scanning laser ophthalmoscope for different pupil entry locations.

In what follows we will concentrate on the implementation by Burns et al. ${ }^{6,9}$ Figure 1 sketches the basic idea of the technique. A small retinal patch is illuminated from a small laser spot imaged in the plane of the eye's pupil. Most of the light is absorbed by melanin, blood, and other ocular pigments. However, some portion of the incident light captured by cones is emitted back toward the pupil, giving rise to a spatially localized, brighter region in the pupillary light distribution. This spatially localized distribution of light returning through the pupil can be fitted to a Gaussian function, characterized by the shape factor $\rho$. Another portion of the incident light is multiply scattered in different retinal and subretinal structures and is reflected back diffusely, filling the entire pupil and appearing in the distribution as a spatially uniform background.

The reflectometric technique has been thought of as a sort of objective version of the psychophysical StilesCrawford effect (SCE) (in fact, Delint et al. ${ }^{12}$ refer to their reflectometric measurements as optical $\mathrm{SCE}^{12}$ ) for several reasons: (1) That is, photoreceptors act as fiber optics that guide light from the pupil into the cone outer segment. This waveguiding produces the directional sensitivity measured psychophysically as the SCE. If the cone photopigment is bleached, the photoreceptors also redirect light back out of the eye, thus acting as an emitting antenna. This emitted light has an angular dependence similar to the collection angle. (2) The location in the pupil of maximum intensity varies across subjects ${ }^{6}$ but coincides with the peak of the SCE in the same subjects. ${ }^{8}$ (3) The fraction of guided light is maximized when the entry pupil is close to the location of the peak of the reflected light distribution. For these reasons it has been argued that the shape factor $\rho$ represents a measurement of the directionality and alignment of the photoreceptors, although it is known that the reflectometric $\rho$ is narrower than the measured psychophysical Stiles-Crawford $\rho .{ }^{8}$ Van Blockland ${ }^{10}$ and Burns et al. ${ }^{6,8}$ found $\rho$ values of $\sim 0.10 \mathrm{~mm}^{-2}$ in the center of the fovea (Gorrand and Delori $^{11}$ and Delint et al. ${ }^{12}$ found values of $\sim 0.20 \mathrm{~mm}^{-2}$, owing to their double scanning), whereas typical values 


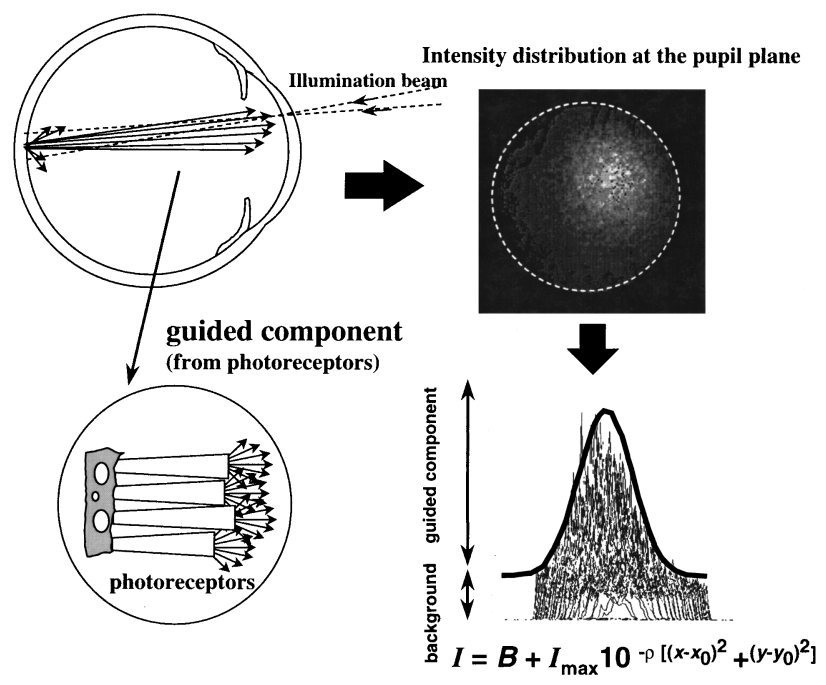

Fig. 1. Schematic representation the reflectometric technique. A small portion of the retina is illuminated in Maxwellian view (top left panel). Part of the light is multiply scattered and reflected back toward the pupil; part is guided along the cones and reradiated back toward the pupil (left panels). The image of the pupil is formed on a cooled CCD camera (top right panel). After the corneal reflexes are blocked, the brightest part of the image corresponds to light reflected back from the cones. The intensity distribution at the pupil plane can be fitted to a constant (background) plus a Gaussian (guided or directed component). The parameters of the fit are $B$ (constant background), $I_{\max }$ (maximum intensity), $x_{0}$ and $y_{0}$ (coordinates of the peak of the distribution), and $\rho$ (shape factor, proportional to the inverse square root of the width of the distribution).

for psychophysical Stiles-Crawford $\rho$ are $\sim 0.05 \mathrm{~mm}^{-2}$ (Ref. 13). The differences between reflectometric and psychophysical $\rho$ are still more marked at higher eccentricities. $^{8}$

Considerable effort has been directed toward understanding the differences between reflectometric and psychophysical Stiles-Crawford measurements. Gorrand and Delori ${ }^{14}$ developed an elegant model based on waveguide theory. Their approach is based (for given diameters and refractive indices of the different segments of a foveal cone) on the number of modes that are guided inside the cones (psychophysical SCE) and on the number of modes that are guided backward (what they call optical SCE). They consider coupling coefficients that account for the interaction of light backscattered at the outersegment-retinal-pigment epithelium interface and recaptured by the cones. Their model predicts a shape factor $\rho$ for reflectometric functions that is twice the shape factor of the Stiles-Crawford function. ${ }^{15}$ It is difficult to evaluate the model for other conditions (i.e., other cone diameters corresponding to other retinal locations). Their model assumes that the waveguide parameter ${ }^{16} \mathrm{~V}$ $<3.832$, so only six modes propagate inside the cone.

Expressions become much more complicated for $V$ $>3.832$, such as would occur for larger cones in the parafovea. ${ }^{17}$ Typically, the predictions from waveguide models (which have been extensively used to explain the psychophysical SCE) are quite sensitive to slight changes of cone diameter, refractive indices, and wavelength. ${ }^{18}$ Presumably, the reflectometric $\rho$ predicted by waveguide models will also show a nonsystematic variation with these parameters.
To model the distribution of light in the pupil plane fully, it is necessary to consider both the waveguide nature of the cones, which serves to shape the angular emission of light from the cones, and the interference of light from different cones, since each cone can act as a small coherent light source, and then light with different phases (due to differences in cone lengths) will add coherently at the pupil plane (far field). In fact, when one looks at the images of the pupil digitized by Burns et al. ${ }^{6}$ the speckle structure is readily apparent. This speckle is reminiscent of the speckle patterns produced by light scattered from rough surfaces.

In this manuscript we will first outline the effect of scattering alone on light reflected from any rough surface that reasonably approximates the dimensions of the cones. We will then show that scattering alone can cause changes in the measured distribution of light in the plane of the pupil as a function of retinal location. We will next reintroduce the waveguide analysis, showing that because the waveguide nature of the cones tends to direct light from different cones toward the same pupillary location, the overall distribution of light measured in the pupil is narrower than expected from either scattering or waveguiding considered alone.

\section{MODEL AND COMPUTER SIMULATION (SCATTERING COMPONENT)}

We treat the cones as cylinders inserted into a medium of a given index of refraction, approximately equally spaced but with random length variations. When light illuminates this retinal cone mosaic, light emerges from each cone with a different phase than that from adjacent cones, owing to the differences in length. The effect of this assumption is that the spatial distribution of light returning from the cones toward the pupil can be modeled in the same way as light reflected from a rough surface. The total field scattered from a rough surface in a certain direction is the sum of elementary waves scattered in that direction by each elementary scatterer-in our case, by each cone. Therefore waves coming from each photoreceptor at a different phase will add coherently at the pupil plane. In the extreme, unrealistic case that the cones and the interphotoreceptor matrix have the same index of refraction, our model retina would be equivalent to a perfectly polished surface and light would be reflected in a single direction. The case in which all the cones had equal lengths would be approximately the same case as a diffraction grating: Light would be reflected back specularly into the different orders of diffraction. In the case of a locally hexagonal array, this would produce circles or noisy hexagons located at multiples of the characteristic spatial frequency of the array. For the more realistic case of cones with slightly different lengths (randomly distributed), light will scatter into various directions (with some privileged directions receiving more energy than others). For small cone-length differences (a smooth surface), the coherent, specular component (the orders of diffraction) will be more intense than the dif- 
fused component. For large cone-length differences (rough surface), the coherent component will be masked by the diffused component.

In the following sections we describe the parameters that characterize a random surface, the scattering theory on which we base our model, the range of applicability of this theory, the additional assumptions that we adopt to model the cones, and the computer simulations of the light intensity at the pupil plane.

\section{A. Scattering Theory: Applicability of the Kirchhoff Approximation}

The scattering of electromagnetic waves from rough surfaces has been extensively studied both theoretically (see Refs. 19 and 20 for a review) and experimentally. ${ }^{21}$ It has been shown that characteristics of a random surface determine the properties of the electromagnetic field scattered by it. ${ }^{22}$ Scattering theory has even been applied in physiological optics to determine the roughness of the anterior lens surface and other structures. ${ }^{23}$

One widely used formalism for describing scattering is that of Beckmann and Spizzino, ${ }^{24}$ which uses the socalled Kirchhoff approximation. In this method, the field and its derivative are approximated by the tangent to the surface at each point. Thus this approximation neglects both shadowing effects and multiple scattering. In this approximation, only the far-field intensity distribution is calculated (that is, the electromagnetic waves are treated as planar). The theory as first formulated was scalar (no polarization effects were considered) and applied only to perfect conductive surfaces. However, it has been extended to describe any polarization ${ }^{25}$ and to dielectric surfaces. ${ }^{26}$ There are two properties that characterize a rough surface: its spatial scale (correlation length) and its roughness. The Kirchhoff approximation is generally applied to statistically homogeneous and isotropic random rough surfaces with Gaussian statistics and a Gaussian correlation function: $\quad C(\tau)=\exp \left(-\tau^{2} / T^{2}\right)$, where $T$ is the correlation length, namely, the distance between "valleys and hills" of the surface. The roughness is defined as the standard deviation of the surface heights $\sigma$. Early studies considered that the approximation failed for $T \lesssim \lambda$ and for $\sigma / T>\lambda .{ }^{27}$ A recent study, ${ }^{28}$ based on the extinction theorem, established that the Kirchhoff approximation held over a broader range, depending on the relationship between $T, \sigma$, and $\lambda$ and the angle of incidence. For example (see Fig. 11 in Ref. 28), for $T=0.4 \lambda$ the Kirchhoff approximation is valid if $\sigma$ $<0.15 T$ and for $T>2 \lambda$ if $\sigma<0.3 \lambda$ for normal incidence. In principle, scattering theory (Kirchhoff as well as other solutions) was developed for surfaces with gentle slopes. Nevertheless, Deryugin ${ }^{29}$ showed that the Kirchhoff approximation was still accurate for surface profiles with sharp edges, at least for the conditions $\Lambda>1.9 \lambda$ and $\Lambda / w \sim 4$, where $\Lambda$ and $w$ are the period and the radius, respectively, of the rectangular corrugation.

In general, the incoherent part of the mean scattered power (also known as the diffused component) is proportional to $\exp \left(-\pi^{2} T^{2} r^{2} / \lambda^{2} z^{2}\right)$ at normal incidence (and can be approximated by this quantity at small angles of incidence), $r$ is the distance to the center of the intensity dis- tribution in the plane of observation, and $z$ is the distance between the surface and the plane of observation. ${ }^{30}$

\section{B. Retinal Mosaic Model}

To apply scattering theory to the retinal cone mosaic, we modeled the cone mosaic similar to that of Marcos and Navarro, ${ }^{31}$ although for the present work we consider larger retinal patches $(0.68 \mathrm{deg})$. To generate the model mosaic, we digitized a photomicrograph of a transverse section of an excised fovea from Curcio et al. ${ }^{32}$ and produced a dot lattice with the location of the dots at the cone centers. The scale at the retinal image plane was set at $0.77 \mu \mathrm{m} /$ pixels, which produced a scale of $0.0457 \mathrm{~mm} /$ pixel at the eye's pupil plane for $256 \times 256$ images. The cone array was scaled to achieve the desired row-to-row cone spacing $s$ for different retinal eccentricities. Since the retinal patches of Curcio et al. ${ }^{32}$ contain only a few cones, we generated a cone array image by tiling the appropriate number of the scaled (reduced) cone array images. To avoid aliasing artifacts, we added spatial jitter (a random-distance, normally distributed $\sigma=0.16 \mathrm{~s}$ ) to the location of the cone centers from the original image. Below, we will consider row-to-row cone distances ranging from 2.25 to $7 \mu \mathrm{m}$ (corresponding to retinal eccentricities from the central fovea to the near parafovea). Although we are aware that with a 0.68 -deg test spot centered at 0 deg, cone spacing varies systematically from the center to the edges (this also happens at larger eccentricities but is less important), for the modeling we assume isotropy in the distribution of the cones within a sample.

The amplitude reflectivity is simulated by convolving the arrays of cones with circles with a radius $40 \%$ of the row-to-row cone spacing (i.e., the cone aperture $d$ is set to
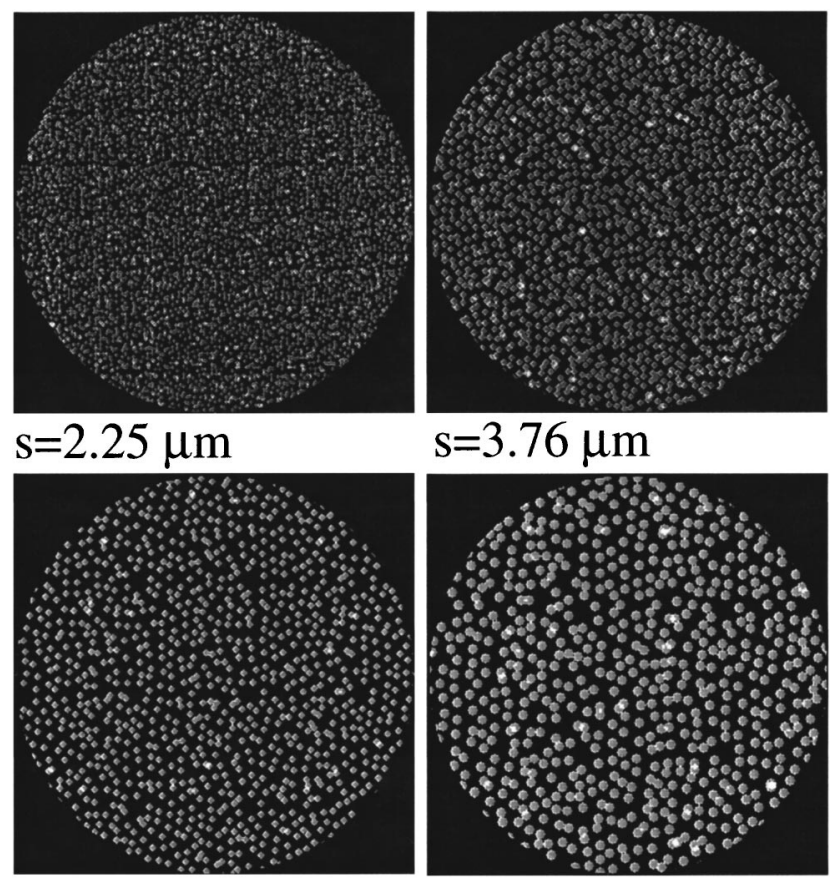

$\mathrm{s}=4.92 \mu \mathrm{m}$
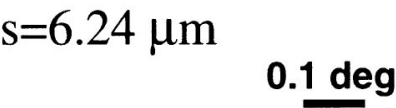

Fig. 2. Examples of simulated cone distributions for four different values of mean cone spacing. 
$80 \%$ of the row-to-row cone spacing $s$ ). Figure 2 shows simulated cone mosaics for four different row-to-row cone spacings.

We assume that random variations of the cone's outersegment lengths produce light emerging from the cones with random phase variations (with Gaussian statistics and zero mean). To our knowledge there are no data available in the literature that suggest a value for the actual variation in cone length, although the portion of the phagocitized outer segment ${ }^{33}$ might give us an idea about the order of magnitude. We have performed computer simulations for random length variations of the outer segment with standard deviations of $0.34,0.51,1.056$, and $1.88 \mu \mathrm{m}$. This corresponds to standard deviations of the effective path-length difference (considering $n_{\mathrm{os}}=1.419$ and $n_{\text {ipm }}=1.347$ for the refractive indices of the cone outer segment and the interphotoreceptor matrix, respectively) of $0.04887,0.073305,0.15204$, and $0.2715 \mu \mathrm{m}$.

As we stated above, the parameters that describe a rough surface are the correlation length $T$ and the roughness standard deviation $\sigma$. The concept of correlation length $T$ is easily defined for surfaces with a Gaussian correlation function: $T$ is basically the radius of the mean scatterer (the distance for which the height is $\mathrm{e}^{-2}$ times the height at its maximum). For our surface with rectangular corrugation (both the length and refractive index of the cone outer segment are assumed to be constant over the aperture of a single cone) we treat $T$ as half the aperture of the cone, $0.5 d$, or, equivalently, $0.4 \mathrm{~s}$. This value is close to the correlation length that we obtain by fitting the correlation function of our cone mosaic (basically a triangular function) to a Gaussian function. Also, we will see that for this correlation length the results of the simulation closely fit Kirchhoff's expressions. Given that, the correlation lengths that we use in our simulation range from 0.9 to $2.8 \mu \mathrm{m}$. Figure 3 shows histograms of the surface effective heights for the four cases considered. The roughness standard deviation stands for the standard deviation of the Gaussian function that fit those histograms.

Our surface parameters fall within the range of applicability of the Kirchhoff approximation and also meet Derugin's $^{29}$ conditions for rectangular corrugations treated under Kirchhoff's approximation (in our notation $\Lambda=s$ and $w=0.5 d$ ). We specifically do not consider any other structure, beneath or below the retinal cone mosaic, although the presence of bodies (i.e., pigmentepithelium cells, melanine granules, etc.) below a scattering surface alters the scattered-light distribution. ${ }^{34}$

\section{Simulation of the Mean Scattered Intensity}

To compare the predictions of scattering theory directly with measurements, we evaluate the mean scattered intensity from the simulated retinal cone mosaic in the plane of the pupil. Our conditions [region of illumination $=0.68 \mathrm{deg}$, distance between object and image planes $=16.7 \mathrm{~mm}$, region of observation limited by the iris, 30 deg] fall within the range of applicability of the Fraunhoffer or far-field approximation. ${ }^{35}$ Thus the complex amplitude at the pupil plane is the Fourier transform of the object. We also checked some of the results by using quadratic wave fronts (Fresnel approximation) instead of

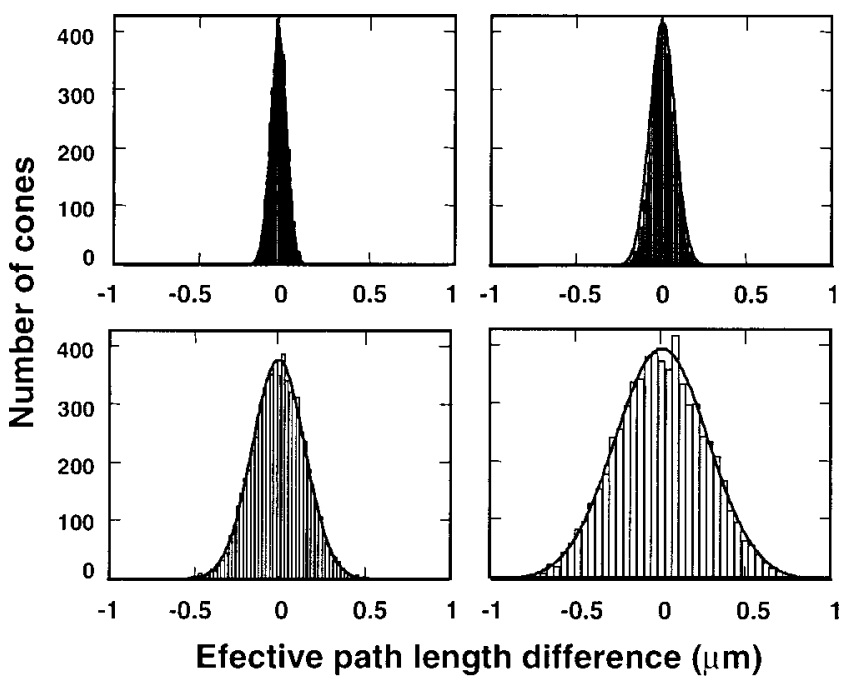

Fig. 3. Histograms of the distribution of effective path-length differences in a simulated cone mosaic in comparison with a Gaussian distribution of the same variance. The roughness is taken as the standard deviation of the distribution. (a) $\sigma$ $=0.04887 \mu \mathrm{m}$, (b) $\sigma=0.0733 \mu \mathrm{m}$, (c) $\sigma=0.152 \mu \mathrm{m}$, (d) $\sigma$ $=0.2715 \mu \mathrm{m}$.

plane waves and found no difference. Since the entry location of the beam never exceeds $4 \mathrm{~mm}$ from the pupil center, we are also close to normal incidence ( $<14 \mathrm{deg})$. Although we assume that the illuminating beam has a Gaussian profile, this does not affect the results. ${ }^{28}$

The amplitude reflectivity of the surface is thus the cone matrix described above, multiplied by a Gaussian function. The phase map is the optical path-length distribution of the cone matrix, multiplied by $2 \pi / \lambda$.

We simulate the intensity distribution at the pupil plane for a short exposure and average 50 images (actual experimental images are typically long exposure). As in a paper by Marcos and Navarro, ${ }^{7}$ we assume that the cone mosaic moves from one exposure to the next, but this affects only the phase distribution (which is changed randomly) but not the amplitude (i.e., no change in the position of the cones is considered).

The resulting mean far-field intensity is the sum of two terms, a coherent component and a diffused component. The mean far-field intensity can be expressed as $\left\langle\left|U_{i}\right|^{2}\right\rangle$ $=\left|\left\langle U_{i}\right\rangle\right|^{2}+$ diffused component, where $\langle\cdot\rangle$ stands for the average across realizations and $U_{i}=\operatorname{FFT}\left(O_{i}\right), O_{i}$ is the instantaneous complex amplitude of the retinal reflectivity. Note that the coherent component is not accessible experimentally with conventional imaging means, since it requires measurement of both the modulus and the phase for each flash. The relation between the diffused and the coherent components changes with roughness. Figure 4 shows all three terms for a cone mosaic with different degrees of roughness and a $3.76-\mu \mathrm{m}$ cone spacing. The first column represents the mean far-field intensity, the second column the coherent component, and the third column the diffused component. In the first three rows the specular peak at the central location has been clipped to enhance the contrast. Each image has been normalized to its highest intensity. For slight roughness, the coherent component almost masks the diffused component of the scattered intensity. Since our surface is nearly peri- 


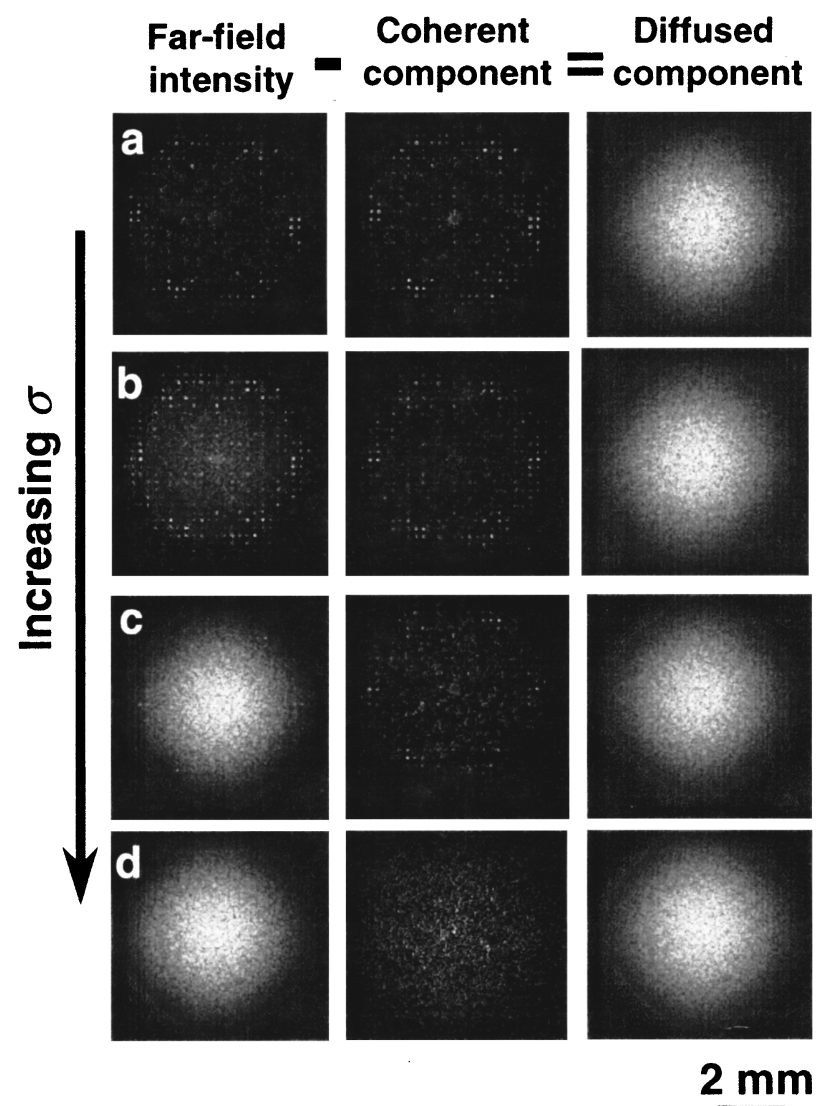

Fig. 4. Far-field intensity, coherent component, and diffused component for a simulated 0.68-deg cone mosaic with $3.76 \mu \mathrm{m}$ of mean cone spacing for different degrees of roughness. First column, average of intensities; second column, square modulus of the average of complex amplitudes; third column, difference of the first and the second (diffused component). Averages are performed over 50 realizations. The specular peak has been removed in the far-field component and the coherent component in the three first cases. Roughness increases, from top to bottom: (a) $\sigma=0.04887 \mu \mathrm{m}$, (b) $\sigma=0.0733 \mu \mathrm{m}$, (c) $\sigma=0.152 \mu \mathrm{m}$, (d) $\sigma=0.2715 \mu \mathrm{m}$. The coherent component is prominent for slight roughness and is masked by the diffused component for higher roughness. The normalized diffused component (third column) is independent of roughness.
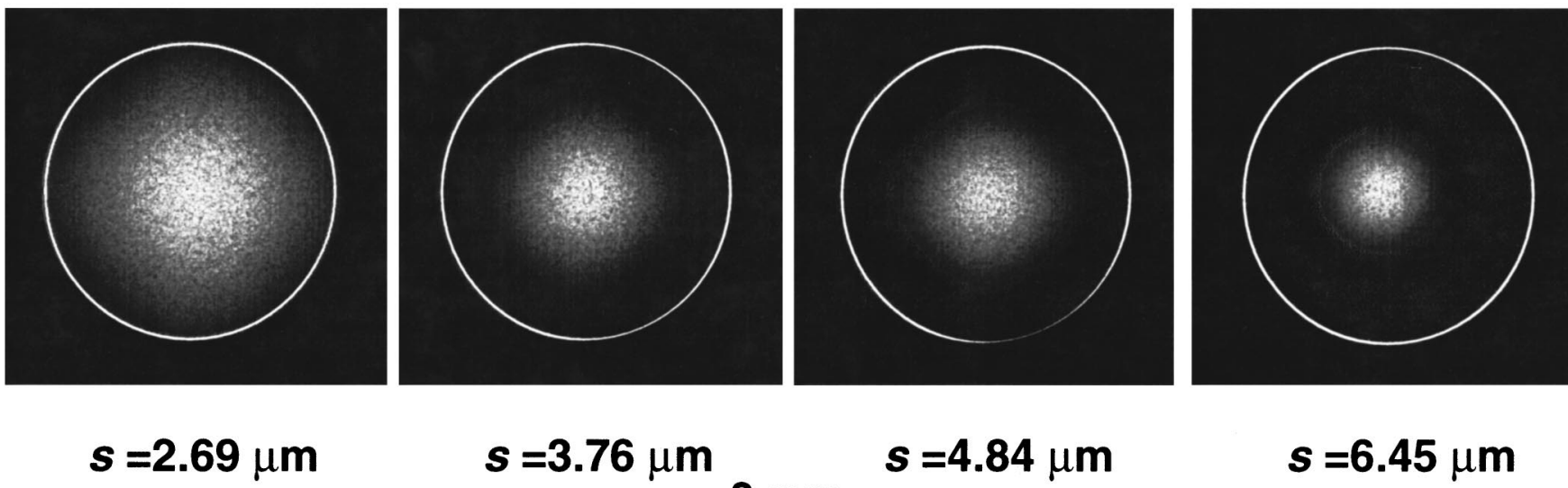

odic, the coherent component consists of a specular peak (which is not in the image) and the first order of diffraction (Yellot's ring ${ }^{36}$ ) of the cone mosaic. As roughness increases, the diffused component increasingly masks the coherent component in the mean scattered intensity. For the roughest surface considered, no coherent specular peak is evident. After normalization the diffused component (third column) is identical for all cases (i.e., the width of the diffused component does not depend on roughness).

The variation in the shape of the diffused component with cone spacing is depicted in Fig. 5 . As the cone spacing increases (from 2.25 to $6.45 \mu \mathrm{m}$ in Fig. 5), the diffused component narrows. As shown in Fig. 6, the diffused component can be fitted to a Gaussian function of the form $10^{-\rho r^{2}}$, where $\rho$ is the shape factor (smaller for broader curves and, as suggested by Figs. 4 and 5, dependent only on cone spacing) and $r$ is the distance from the center of the intensity distribution. We will use the same notation used in the SCE (decimal logarithm) instead of the classical notation in physical optics (natural logarithm) that we have used so far in this paper. Figure 7 plots the variation of the shape factor with cone spacing. The circles are results from the simulation, and the dashed curve is the prediction from Kirchhoff theory: According to Kirchhoff's expression for the diffused component (Subsection 2.A), and using our own notation, we obtain

$$
\rho_{\text {scatt }}=\frac{\pi^{2}(0.4 s)^{2}}{f^{2} \lambda^{2} \ln 10},
$$

with $\lambda=0.543 \mu \mathrm{m}, f=16.7 \mathrm{~mm}$, and $\rho_{\text {scatt }}=0.00844$ $\mathrm{s}^{2}$. The data plotted in Fig. 7 were obtained for $\sigma$ $=0.04788$, illumination area $=0.68 \mathrm{deg}$, averaging of 50 realizations, but we have seen that changing these parameters does not modify $\rho$. To confirm that this spatial limitation at the pupil plane is due only to the phase variation, we calculated the spatial distribution at the pupil plane when there is no modulation in the modulus of the reflectivity and obtained the same result. $s=4.84 \mu \mathrm{m}$ $s=6.45 \mu \mathrm{m}$

\section{$2 \mathrm{~mm}$}

Fig. 5. Simulated diffused component for different values of row-to-row cone spacing. The distribution becomes narrower for increasing cone spacing. 


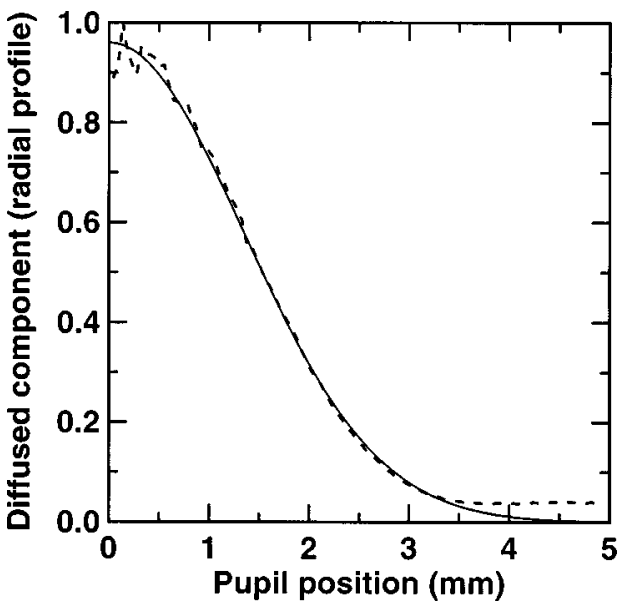

Fig. 6. Fit of the diffused (scattered) component to a Gaussian of the form $10^{-\rho r^{2}}$. Dotted curve, radial profile of the simulated diffused component from a cone mosaic with a mean row-to-row cone spacing of $3.76 \mu \mathrm{m}$; solid curve, fit to a Gaussian ( $\rho$ $=0.11 \mathrm{~mm}^{-2}$ ).

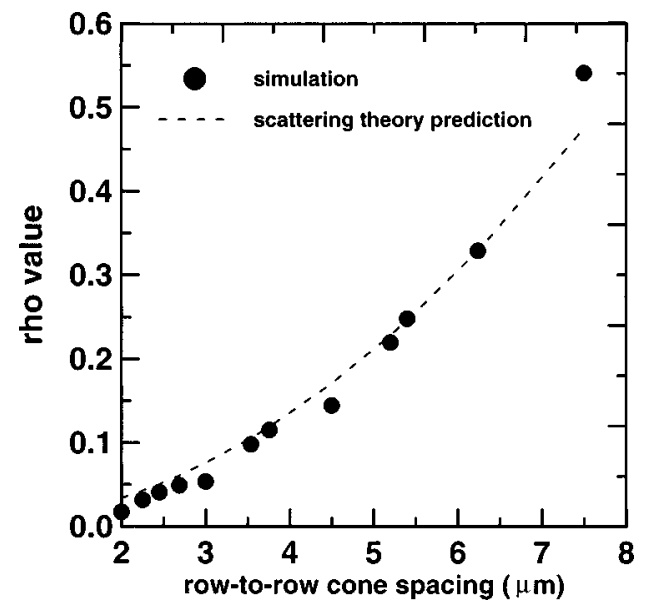

Fig. 7. $\rho$ (obtained by fitting the simulated diffused component as shown in Fig. 6) as a function of row-to-row cone spacing (circles). The dashed curve represents the prediction from scattering theory (Kirchhoff approximation): $\rho=0.00844$ $s^{2}\left(\mathrm{~mm}^{-2}\right)$.

\section{EXPERIMENTAL EVIDENCE FOR SCATTERING}

\section{A. Methods and Subjects}

We used an imaging reflectometer to measure the spatial distribution of the light at the pupil plane returning from the retina. The apparatus and procedure are explained elsewhere (see Refs. 6 and 9 for details). In short, a small patch of the retina ( $1 \mathrm{deg}$ in most experiments, 0.5 deg in a control experiment) is illuminated with a Maxwellian view system by use of a small entry pupil ( 0.18 $\mu \mathrm{m})$. The position of the entrance pupil, and the fixation location (in a separate channel) are under computer control. Light returning from the retina is measured with a cooled high-resolution CCD camera at a plane located conjugate to the subject's pupil. Typically, the exposure time was $1 \mathrm{~s}$, although short-exposure images $(10 \mathrm{~ms})$ were also digitized to improve visibility of speckle (the speckle was blurred in the long-exposure images). Images were $170 \times 170$ pixels, with a scale of $0.08 \mathrm{~mm} /$ pixel at the pupil plane. The directed component of the images is fitted with a Gaussian of the form $I_{\max } 10^{-\rho r^{2}}$, with $r=\left(x-x_{0}\right)^{2}+\left(y-y_{0}\right)^{2}$.

The main difference from the apparatus described in Ref. 6 is the use of three lasers (a 543-nm and a 632-nm $\mathrm{He}-\mathrm{Ne}$ laser and a 670-nm diode laser). The red lasers were added to the optical path by means of a beam splitter and a mirror mounted on a kinematic mount. All the beams are spatially filtered at the same pinhole. Only a single wavelength was used for a single image.

Three normal subjects (SM, JH, and SB), aged 26, 37, and 47 , one female and two males, participated in this study. JH had deuteranomalous color vision. Subjects were dilated with $0.5 \%$ Mydriacil after informed consent was obtained.

\section{B. Short-Exposure Images}

Short-exposure images $(10 \mathrm{~ms})$ were obtained for subject SM in dark-adapted and bleached conditions. Darkadapted images showed a constant background with little modulation. The bleached-state images [Fig. 8(a)] present fully developed speckle, confirming that light returning from the photoreceptors produces speckle patterns. Changing the illumination spot size produces a change in the speckle size (speckle size increases when the spot size is reduced from 1 to $0.5 \mathrm{deg}$ ), as was found for the computer simulations [Fig. 8(b) shows an example for $0.5 \mathrm{deg}]$.

\section{Variation of $\rho$ with Retinal Location}

Burns et al. ${ }^{9}$ showed that $\rho$ values changed rapidly with retinal eccentricity, with the width of the pupil intensity distribution being broader at the center of the fovea than at higher eccentricities. We measured $\rho$ as a function of retinal eccentricity ( 0 deg and $0.5,1,2$, and 3 nasal and temporal) for $543 \mathrm{~nm}$ and compared the results with the predictions from the simulation. Measurements were collected in three different sessions for subjects SM and SB and two for JH. Typically, only a single retinal series is obtained per session at the optimal entry-pupil position (i.e., for which higher maximum intensities are obtained). We found that for subject SM, the optimal entry-pupil lo-

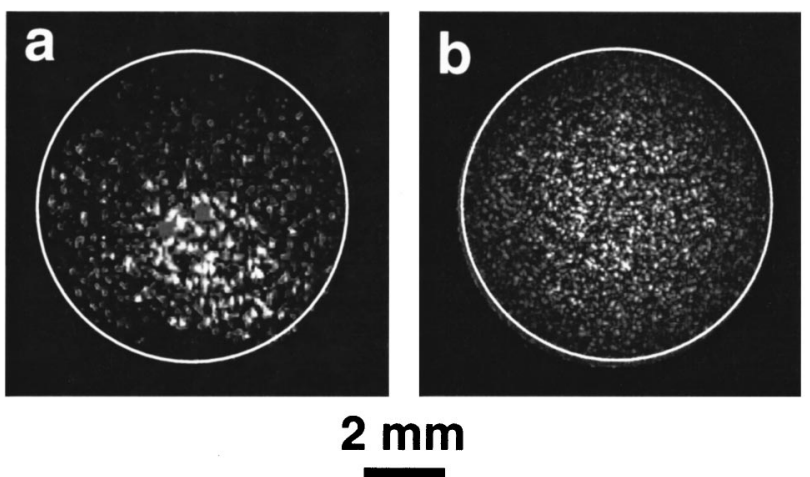

Fig. 8. Short-exposure images at the pupil plane. (a) Experimental (subject SM, 0 deg eccentricity; spot size $0.5 \mathrm{deg}$ ). (b) Simulation (cone spacing $2.25 \mu \mathrm{m}$; roughness $0.271 \mu \mathrm{m}$; illumination spot size: $0.5 \mathrm{deg}$ ). 


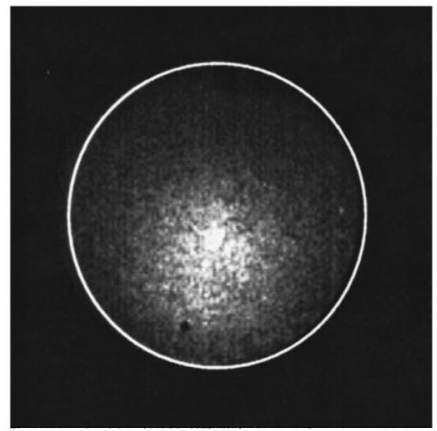

$e=0 \mathrm{deg}$
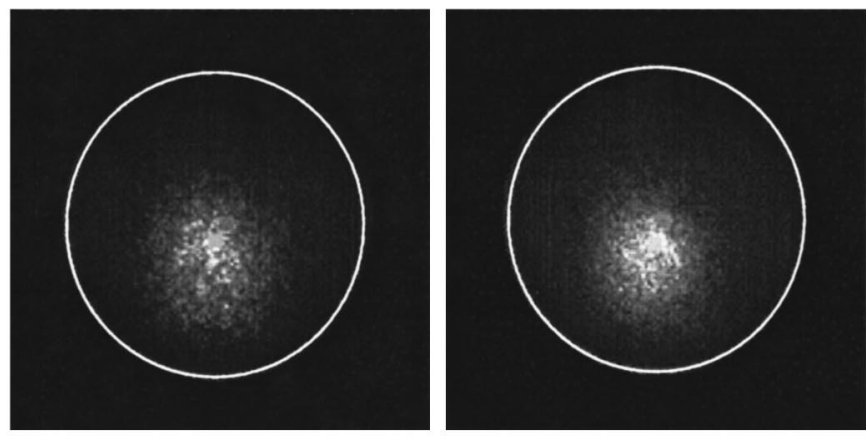

$e=0.5 \mathrm{deg}$

\section{$2 \mathrm{~mm}$}

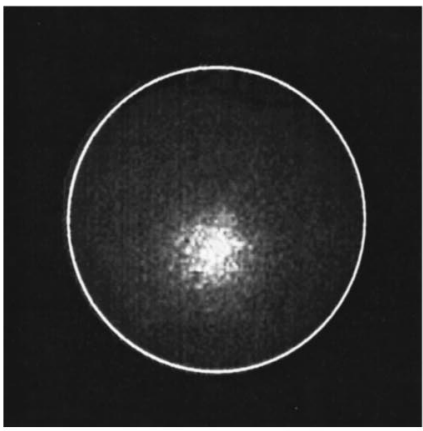

$e=2 \mathrm{deg}$

Fig. 9. Experimental intensity distribution at the pupil plane for different retinal eccentricities in the nasal retina, for subject SM. The corneal reflex has been removed. The distribution narrows as retinal eccentricity (or, equivalently, cone spacing) increases.

cation moved slightly horizontally, as the fixation moved horizontally in the retina. In this case we measured four retinal series in a session by use of entry pupils at $0.5-\mathrm{mm}$ intervals and for each retinal location selected the images with the largest directed component. We have included the results obtained for $3 \mathrm{deg}$, although in some cases at this eccentricity measurements may contain reflection artifacts that could artificially broaden the distribution. We have confirmed that these reflections arise from anterior structures, since they are not diminished under darkadapted conditions, and we have not included data in which these reflections are readily apparent.

Figure 9 shows the intensity distribution at the pupil plane for subject SM at 0, 0.5, 1, and $2 \mathrm{deg}$ (left to right). The results in Fig. 9 are comparable with the simulations of Fig. 5. It is interesting to note that the coherent component does not appear in the experimental intensity distributions, thus suggesting that the photoreceptor array is rough, closer to case (d) in Fig. $4(\sigma=0.27 \mu \mathrm{m})$ than to the more uniform cases.

Figure 10 directly compares the experimental data (for three subjects) with the simulation. We computed the equivalent retinal eccentricity for a given row-to-row cone spacing using data of Curcio et al. ${ }^{32}$ We assumed hexagonal packing and a conversion between millimeters and degrees in the retina of $290 \mu \mathrm{m} / \mathrm{deg}{ }^{37}$ We interpolate the cone spacing data by use of a parabolic fit for retinal eccentricities between 0 and $3.6 \mathrm{deg}$. The conversion is $e$ $=0.05296 s^{2}+0.332087 s-0.0886$, where $e$ is retinal eccentricity in degrees and $s$ is row-to-row cone spacing in micrometers. The solid curve and triangles in Fig. 10 represent the predictions from the simulation. The shape (higher $\rho$ for higher eccentricity) and size of the experimental and the simulated results are similar, up to 2 deg of retinal eccentricity. In the four central degrees, the simulated data are systematically lower than the measured data. Beyond $2 \mathrm{deg}$, the predicted $\rho$ systematically increases, although experimental data do not. This discrepancy between experimental and predicted $\rho$ at higher retinal eccentricities may be due to the presence of rods (which our current model ignores) and larger interphotoreceptor spaces (at higher eccentricity the assumption that the cone aperture is $80 \%$ of the row-to-row spac-

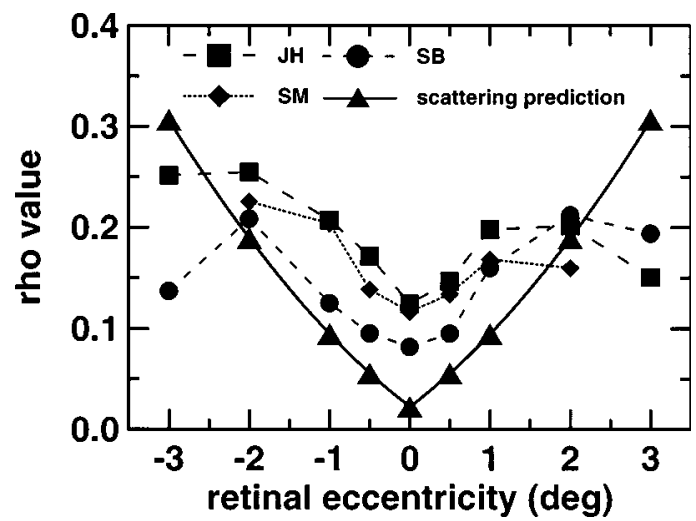

Fig. 10. $\rho$ as a function of retinal eccentricity. Squares, diamonds, and circles represent experimental measurements in a single session for subjects JH, SM, and SB respectively. Triangles are the predictions from the simulation (fitted to Kirchhoff approximation) when a conversion between cone spacing and retinal eccentricity is considered. The illumination wavelength was $543 \mathrm{~nm}$.

ing does not hold). In addition, the presence of artifacts in the images for some of the subjects at higher eccentricities makes the experimental results less reliable. For locations within $2 \mathrm{deg}$ there are two simple reasons for the discrepancy: (1) intersubject variability in cone spacing: For the range of cone densities from the nine eyes studied by Curcio et al. ${ }^{32}$ in the center of the fovea, $\rho$ would range between 0.02 and 0.06 . This explains possible individual discrepancies, although not the shift of the simulated data to a lower value. (2) In the simulations we have assumed that the cone spacing does not vary systematically within the sampled 1-deg region. In fact, cone spacing varies within the region sampled, particularly in the center of the fovea. For the 0-deg location the average cone spacing across $1 \mathrm{deg}$ is lower than the cone spacing at the exact center of the fovea and should produce a higher $\rho$. In Section 4 we will see that a unified model that considers both scattering and waveguides shifts the predictions for $\rho$ at lower retinal eccentricities closer to the experimental data. 


\section{Variation of $\rho$ with Wavelength}

Waveguide models predict a nonsystematic variation of $\rho$ as a function of wavelength. ${ }^{38}$ In contrast, scattering predicts that $\rho$ is inversely proportional to $\lambda^{2}$. As Beckmann and Spizzino point out (Ref. 24, p. 92), "increasing the wavelength has the same effect as decreasing the roughness parameter $\sigma$ and the correlation distance $T$ (or equivalently $s)$." That is, increasing wavelength from $\lambda_{1}$ to $\lambda_{2}$ will decrease $\rho$ by a $\left(\lambda_{2} / \lambda_{1}\right){ }^{2}$

Figure 11(a) shows $\rho$ as a function of eccentricity for the three wavelengths for subject $\mathrm{JH}$, and Fig. 11(b) shows the mean ratio $\rho_{632} / \rho_{543}$ and $\rho_{670} / \rho_{543}$ (averaging across subjects and sessions). The straight lines represent the ratio predicted by scattering theory for both ratios $(0.738$ and 0.657 , respectively). In addition to the eccentricity series, we performed multiple, repeated measurements at 0 and 2 deg nasal, both at the optimal pupil-entry position and at other pupil positions. Table 1 shows the average of $\rho$ ( 0 and $-2 \mathrm{deg})$ for each subject and wavelength (averaging across sessions) along with the standard deviation of the measurement. The last two rows represent $\rho_{632} / \rho_{543}$ and $\rho_{670} / \rho_{543}$, respectively, with the estimated error of the ratio. In general, there is more variability in the data as we increase wavelength. There are two main reasons for this: (1) For longer wavelengths, there is a much higher contribution of light returning from deeper structures, ${ }^{39}$ so the ratio of the guided component to the background decreases. Since the signal-to-noise ratio decreases, there is more variability in the fit. (2) We have observed that measurements with red light are more sensitive to defocus in the retinal plane than are measurements in green light. Thus part of the variability may be caused by slight changes in focus from session to session. Also, variability for subject $\mathrm{SB}$ is higher than for the other two subjects, whereas the results for subject $\mathrm{JH}$ are quite stable. In general, the ratios $\rho_{\text {red }} / \rho_{543}$ are slightly higher than the prediction from the scattering theory.

\section{UNIFIED WAVEGUIDE-SCATTERING MODEL}

In Section 2 we modeled the effect of phase variations of light emitted from the cones on the spatial intensity distribution at the pupil plane, and in Section 3 we showed that actual measurements of the distribution of light in the pupil are qualitatively similar to the predictions of the scattering model. We have seen that a spatially limited intensity distribution is a natural consequence of the scattering model. However, in actuality light coming from the retinal cones cannot interfere over the entire pupil. The waveguide nature of the cone photoreceptors limits the area over the pupil where the interference can take place by limiting the angular extent of the interfering waves. For sufficiently small cone apertures (small enough to be treated as coherent sources), as may be the case for cones at the center of the fovea, ${ }^{9}$ this angular extent is limited by diffraction at the cone inner segment (or myode anterior face). Diffraction sets a minimum limit for the angular extent. Waveguide models ${ }^{18,14}$ approximate the angular extent into which a cone radiates light by a Gaussian function (with a shape factor that we denote by $\rho_{\mathrm{wg}}$ ).
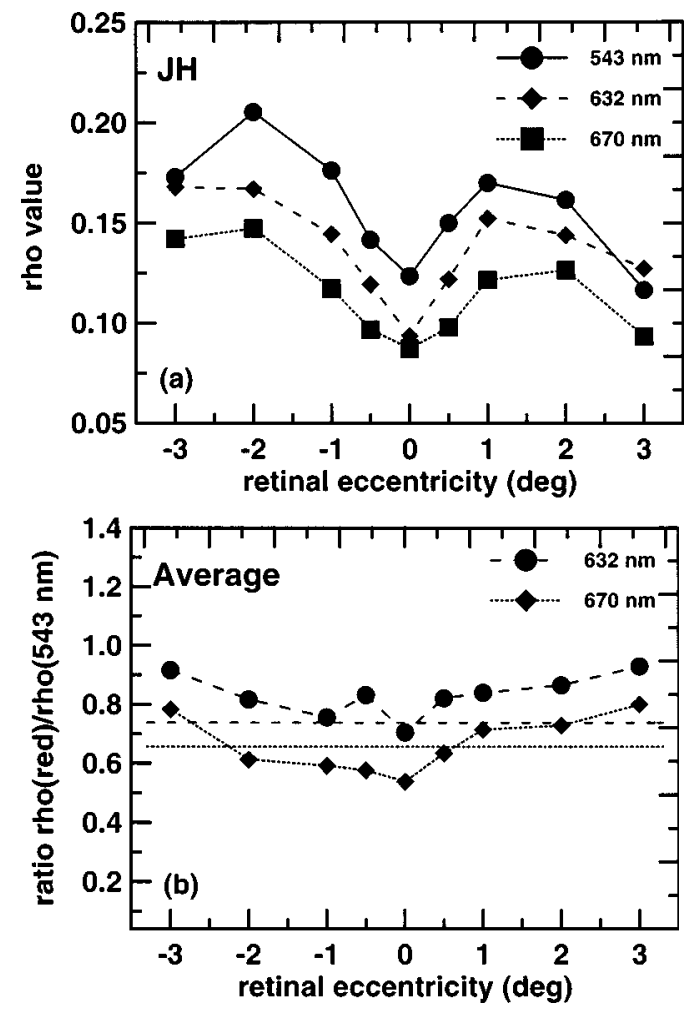

Fig. 11. (a) Variation of $\rho$ with retinal eccentricity for three different wavelengths $(543,632$, and $670 \mathrm{~nm}$ ) for subject $\mathrm{JH}$ (average across three sessions). (b) Ratios $\rho_{632} / \rho_{543}$ (dashed curve, circles) and $\rho_{670} / \rho_{543}$ (dotted curve, diamonds), averaging across the three subjects and all sessions. The dashed horizontal line represents the scattering prediction for $\rho_{632} / \rho_{543}$ and the dotted line for $\rho_{670} / \rho_{543}$.

Table 1. Ratios $\rho_{632} / \rho_{543}$ and $\rho_{670} / \rho_{543}$ for Three Subjects and Two Retinal Eccentricities ${ }^{a}$

\begin{tabular}{|c|c|c|c|c|c|c|c|}
\hline \multirow{2}{*}{$\begin{array}{c}\rho \text { Ratio } \\
( \pm \mathrm{std} / 2)^{b}\end{array}$} & \multicolumn{2}{|c|}{ JH } & \multicolumn{2}{|c|}{ SM } & \multicolumn{2}{|c|}{ SB } & \multirow{2}{*}{$\begin{array}{l}\text { Scattering } \\
\text { Prediction }\end{array}$} \\
\hline & $0 \mathrm{deg}$ & $-2 \mathrm{deg}$ & $0 \mathrm{deg}$ & $-2 \mathrm{deg}$ & $0 \mathrm{deg}$ & $-2 \mathrm{deg}$ & \\
\hline$\rho_{632} / \rho_{543}$ & $\begin{array}{c}0.7933 \\
( \pm 0.0334)\end{array}$ & $\begin{array}{c}0.8146 \\
( \pm 0.0235)\end{array}$ & $\begin{array}{c}0.7783 \\
( \pm 0.0411)\end{array}$ & $\begin{array}{c}0.7740 \\
( \pm 0.0814)\end{array}$ & $\begin{array}{c}0.7661 \\
( \pm 0.10)\end{array}$ & $\begin{array}{c}0.7368 \\
( \pm 0.0816)\end{array}$ & 0.7381 \\
\hline$\rho_{670} / \rho_{543}$ & $\begin{array}{c}0.7764 \\
( \pm 0.0418)\end{array}$ & $\begin{array}{c}0.7523 \\
( \pm 0.0126)\end{array}$ & $\begin{array}{c}0.5132 \\
( \pm 0.0024)\end{array}$ & $\begin{array}{c}0.6015 \\
( \pm 0.05)\end{array}$ & & & 0.6568 \\
\hline
\end{tabular}

${ }^{a}$ Averages across at least three sessions for $632 \mathrm{~nm}$ and two sessions for $670 \mathrm{~nm}$.

${ }^{b}$ std $=$ standard deviation. 
There are two major implications when we combine waveguide properties and cone directionality in our model:

1. The mirror laws do not apply here. The experimental observation is that the peak of the intensity distribution always appears in the same position in the pupil, no matter the angle of incidence. Thus, whereas the maximum intensity changes in magnitude with the angle of incidence, the location does not. In summary, the backscattered beam goes along a specific direction regardless of the angle of incidence. We believe that this specific location in the pupil corresponds to the location of the axes of the photoreceptors for two reasons. First, the intensity of the guided component is maximum when the entry pupil coincides with the location of the peak. Second, the location of the peak in reflectometric measurements coincides with the location of the psychophysical Stiles-Crawford peak. ${ }^{8}$ Although scattering does not take place as in a conventional reflection from a standard rough surface, for which the light is reflected and reemitted about the specular direction, this is not incompatible with the model. Instead, the light is captured by the photoreceptors (acting as waveguides), guided along the inner segments, and emitted back along the photoreceptor axis toward the pupil and not along the direction of specular reflections.

2. Since both the scattering and the waveguide components go along the same direction, the resulting distribution is the multiplication of two Gaussian functions with shape factors $\rho_{\text {scatt }}$ and $\rho_{\text {wg }}$, respectively. ${ }^{40}$ The combined intensity distribution is predicted by this unified model (based on scattering and waveguides) to be Gaussian and to have a shape factor $\rho_{\text {scatt }}+\rho_{\mathrm{wg}}$. This resulting distribution will always be narrower than the intensity distribution predicted by scattering or waveguide components alone. Therefore the predicted $\rho$ in Fig. 11 is shifted toward higher values, closer to the experimental data (for eccentricities up to $2 \mathrm{deg}$ ). The extent of the correction for waveguides depends on the relation between $\rho_{\text {scatt }}$ and $\rho_{\text {scatt }}+\rho_{\text {wg }}$. Also, ratios $\rho_{\text {red }}$ to $\rho_{543}$ (Table 1) with respect to the ratios predicted by pure scattering slightly change with the introduction of a waveguide correction.

Unfortunately there are no published data on the variation of the acceptance angles of single cones predicted by waveguide models. The models of Snyder and Pask $^{18}$ and Gorrand and Delori ${ }^{14}$ apply only to foveal cones with specific diameters. We have made simple computations based on Snyder and Pask's ${ }^{18}$ model for cones at different retinal eccentricities, using Curcio's ${ }^{41}$ cone inner segment diameters. We have used more recent data for indices of refraction data than those used by Snyder and Pask, ${ }^{18}$ although in this case, the differences in the predictions caused by the change of indices of refraction are small (only $\sim 4.5 \%$ ). The long-dashed curve in Fig. 12 shows our predictions for $\rho$ from the waveguide model: $\rho$ increases from approximately 0.05 to 0.08 when moving from 0 to $3 \mathrm{deg}$. If we assume that there is no significant photoreceptor disarray at these eccentricities ${ }^{14,42}$ and ignore effects such as cross talk and self-screening, we can compare the angular tuning of a single cone with the psychophysical Stiles-Crawford function. Measurements ${ }^{43,44}$ indicate that the StilesCrawford directionality ( $\rho$ factor) increases from $\sim 0.05$ on average at the center of the fovea to 0.08 at $3 \mathrm{deg}$, in good agreement with the predictions from the waveguide model.

The predictions for $\rho$ as a function of retinal eccentricity (for $\lambda=543 \mathrm{~nm}$ ) from the unified model (scattering + waveguide) are represented in Fig. 12 by the dotted curve. We observe that the effect of adding the waveguide component (long-dashed curve) is to shift the whole curve predicted by scattering (dashed curve) toward higher values, although the major behavior is already given by the scattering model. In Fig. 12 we also include the model proposed by Burns et al. ${ }^{9}$ (see Fig. 7 in Ref. 9), assuming that, at the foveal center, the cones act as simple diffraction-limited apertures. Circles represent an average (across subjects and sessions) of the experimental measurements of $\rho$ for $\lambda=543 \mathrm{~nm}$, adjusted to account for the average eccentricity of cones within the measurement. As we mentioned above, at higher eccentricities the discrepancy may be explained by the presence of rods, which act as a secondary photoreceptor mosaic with different scattering properties, and the possible relative narrowing of the cones with respect to the row-torow spacing (that is, the cone aperture may no longer be $80 \%$ of cone row-to-row spacing).

Since predictions of waveguide models for $\rho$ as a function of wavelength are quite sensitive to the exact knowledge of cone dimensions and indices of refraction, ${ }^{38}$ they are not useful in deriving the ratio $\rho_{\text {red }}$ to $\rho_{543}$ from the compound model. The prediction from pure scattering agreed roughly with the experimental findings, and the correction for the appropriate cone tuning should give a closer agreement.

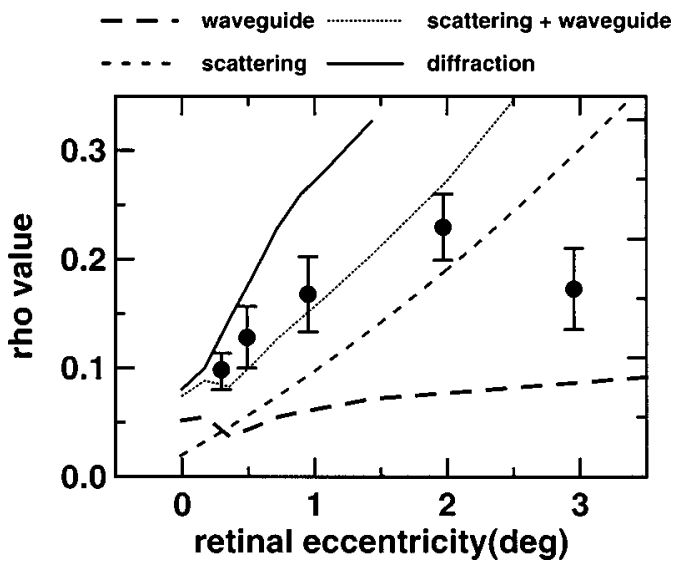

Fig. 12. Predictions of different models for the variation of $\rho$ as a function of retinal eccentricity in comparison with the experimental measurements. Long-dashed curves, prediction of waveguide theory (which we have derived as an extension of the Snyder and Pask model ${ }^{17}$ ); Short-dashed curve, prediction from scattering theory; Dotted curve, sum of the two previous curves (scattering model including the waveguide properties of the photoreceptors); solid curve diffraction limit for apertures of the size of the cone inner segments (see Burns et $a l .{ }^{9}$ ); circles, average experimental measurements (across subjects and sessions). Data have been shifted to the eccentricity equivalent to the mean cone spacing within the sample (the shift is appreciable only for the lower eccentricity: $0 \mathrm{deg}$ is equivalent to $0.3 \mathrm{deg}$ ). Error bars represent the standard deviation. The illumination wavelength for measurements and models was $543 \mathrm{~nm}$. 


\section{CONCLUSIONS}

In conclusion, the unified model that we propose, based on a combination of scattering and waveguides, accounts for the results obtained experimentally in reflectometric measurements: the narrowing of the intensity distribution at the pupil plane as a function of retinal eccentricity and the broadening of the distribution for increasing wavelength. Reflectometric measurements provide information regarding both the waveguide properties of the photoreceptors and their spacing (implicit in the expressions for the scattering component). An adequate extraction of the waveguide component may lead to automatic knowledge of the cone spatial distribution, or, inversely, the knowledge of the spatial distribution of the cones in our observers would potentially allow us to extract the pure waveguide properties of their photoreceptors in vivo.

\section{ACKNOWLEDGMENTS}

We wish to thank J. A. Sánchez-Gil and J. M. Soto-Crespo for their comments on scattering in the earlier stages of this work. This work was supported by the National Institutes of Health grant EY-04395, the Chartrand Foundation, and the Massachusetts Lions Eye Research Foundation. In addition, S. Marcos was supported by a MEC/ Fulbright Postdoctoral Fellowship, Ministerio de Educación y Cultura, Spain, and J. C. He by National Research Service Award grant EY-06629.

\section{REFERENCES AND NOTES}

1. J. Santamaría, P. Artal, and J. Bescós, "Determination of the point-spread function of human eyes using a hybrid optical-digital method," J. Opt. Soc. Am. A 4, 1109-1114 (1987).

2. A. E. Elsner, S. A. Burns, G. W. Hughes, and R. H. Webb, "Reflectometry with a scanning laser ophthalmoscope," Appl. Opt. 31, 3697-3710 (1992).

3. A. E. Elsner, S. A. Burns, and R. H. Webb, "Mapping cone pigment optical density in humans," J. Opt. Soc. Am. A 10, 52-58 (1993).

4. D. R. Williams, D. H. Brainard, M. J. McMahon, and R. Navarro, "Double-pass and interferometric measures of the optical quality of the eye," J. Opt. Soc. Am. A 11, 3123-3135 (1994).

5. S. Marcos, R. Navarro, and P. Artal, "Coherent imaging of the cone mosaic in the living human eye," J. Opt. Soc. Am. A 13, 897-905 (1996).

6. S. A. Burns, S. Wu, F. C. Delori, and A. E. Elsner, "Direct measurement of human cone-photoreceptor alignment," J. Opt. Soc. Am. A 12, 2329-2338 (1996).

7. S. Marcos and R. Navarro, "Determination of the foveal cone spacing by ocular speckle interferometry: limiting factors and acuity predictions," J. Opt. Soc. Am. A 14, 731740 (1997).

8. S. A. Burns, A. E. Elsner, J. M. Gorrand, M. R. Kreitz, and F. C. Delori, "Comparison of reflectometric and psychophysical measures of cone orientation," in Noninvasive Assessment of the Visual System Vol. 1 of 1992 OSA Technical Digest Series (Optical Society of America, Washington, D.C., 1992), pp. 160-163; J. He and S. A. Burns, "Cone photoreceptor directionality assessed using psychophysical and imaging techniques in vivo," Invest. Ophthalmol. Visual Sci. Suppl. 38, 1015 (1997).

9. S. A. Burns, S. Wu, J. C. He, and A. E. Elsner, "Variations in photoreceptor directionality across the central retina," J. Opt. Soc. Am. A 14, 2033-2040 (1997).

10. G. J. Van Blockland, "Directionality and alignment of the foveal photoreceptors assessed with light scattered from the human fundus in vivo," Vision Res. 26, 495-500 (1986).

11. J. M. Gorrand and F. C. Delori, "A reflectometric technique for assessing photoreceptor alignment," Vision Res. 35, 999-1010 (1995).

12. P. J. Delint, T. T.-J. M. Berendschot, and D. van Norren, "Local photoreceptor alignment measured with a scanning laser ophthalmoscope," Vision Res. 37, 243-248 (1997).

13. R. A. Applegate and V. Lakshminarayanan, "Parametric representation of Stiles-Crawford functions: normal variation of peak location and directionality," J. Opt. Soc. Am. A 10, 1611-1623 (1993).

14. J. M. Gorrand and F. C. Delori, "A model for assessment of cone directionality," J. Mod. Opt. 44, 473-491 (1997).

15. The factor of 2 is for the design of Refs. 6 and 9 Burns et al. For their own design the prediction is a factor of 4 .

16. $V=\pi D\left(n_{\text {co }}^{2}-n^{2}\right)^{1 / 2} / \lambda$; where $D$ is the diameter of the waveguide, and $n_{\text {co }}$ and $n$ are the respective refractive indices of the core and surrounding medium; E. Snitzer, "Cylindrical dielectric waveguide modes," J. Opt. Soc. Am. 51, 491-498 (1961).

17. For those larger cones the intensity distribution at the pupil plane becomes the sum of 1296 terms, instead of 81 . J. M. Gorrand, Faculté de Medicine, Inserm U.684 BP 38 Clermont-Ferrand 63001, France (personal communication, 1997).

18. A. W. Snyder and C. L. Pask, "The Stiles-Crawford effectexplanation and consequences," Vision Res. 13, 1115-1137 (1973).

19. M. Nieto-Vesperinas and J. C. Dainty, eds., Scattering in Volumes and Surfaces (North-Holland, Amsterdam, 1990).

20. M. Nieto-Vesperinas, Scattering and Diffraction in Physical Optics (Wiley, New York, 1991), Chap. 7.

21. K. A. O'Donnell and E. R. Méndez, "Experimental study of scattering from characterized random surfaces," J. Opt. Soc. Am. A 4, 1194-1205 (1987).

22. J. M. Bennett, ed., Surface Finish and Its Measurement, Vol. 2 of Collected Works in Optics (Optical Society of America, Washington, D.C., 1992).

23. D. Miller and G. Bennedek, Intraocular Light Scattering: Theory and Clinical Applications (Thomas, Springfield, Ill., 1973); R. Navarro, "Incorporation of intraocular scattering in schematic eye models," J. Opt. Soc. Am. A 2, 1981-1984 (1985). R. Navarro, J. Méndez-Morales, and J. Santamaría, "Optical quality of the eye lens surfaces from roughness and diffusion measurements," J. Opt. Soc. Am. A 3, 228234 (1996).

24. P. Beckmann and A. Spizzino, The Scattering of Electromagnetic Waves from Rough Surfaces (Pergamon, New York, 1963).

25. M. Nieto-Vesperinas, "Depolarization of electromagnetic waves scattered from slightly rough random surfaces: a study by means of the extinction theorem," J. Opt. Soc. Am. 72, 539-547 (1982).

26. J. A. Sánchez-Gil and M. Nieto-Vesperinas, "Light scattering from random rough dielectric surfaces," J. Opt. Soc. Am. A 8, 1270-1286 (1991).

27. M. Nieto-Vesperinas and N. García, "A detailed study of the scattering of scalar waves from random rough surfaces," Opt. Acta 28, 1651-1672 (1981).

28. J. M. Soto-Crespo and M. Nieto-Vesperinas, "Electromagnetic scattering from very rough surfaces and deep reflection gratings,” J. Opt. Soc. Am. A 6, 367-384 (1989).

29. L. N. Deruyugin, "Equations for the coefficients of reflection of waves from a periodically rough surface," Dokl. Akad. Nauk SSSR 87, 913-916 (1952).

30. The diffused component can be approximated by $\exp \left(-Q^{2} T^{2} / 4\right)$, where $Q$ is the momentum transfer of the surface (see Refs. 24 and 25). At normal incidence $Q=(2 \pi / \lambda) \sin \theta$, where $\theta$ is the angle of scattering (called, the observation angle), and $\sin \theta=r / z$.

31. S. Marcos and R. Navarro, "Imaging the foveal cones in vivo through ocular speckle interferometry: theory and numerical simulations," J. Opt. Soc. Am. A 13, 2329-2340 (1996). 
32. C. A. Curcio, K. R. Sloan, R. E. Kalina, and A. E. Hendrickson, "Human photoreceptor topography," J. Comp. Neurol. 292, 497-523 (1992).

33. R. Young, "The renewal of rod and cone outer segments in the rhesus monkey," J. Cell Biol. 39, 303-318 (1971).

34. A. Madrazo and M. Nieto-Vesperinas, "Scattering of light and other electromagnetic waves from a body buried beneath a highly rough random surface," J. Opt. Soc. Am. A 14, 1859-1866 (1997).

35. J. W. Goodman, Introduction to Fourier Optics (McGrawHill, New York, 1968).

36. J. J. Yellot, "Spectral analysis of spatial sampling of photoreceptors: topological disorder prevents aliasing," Vision Res. 22, 1205-1210 (1982).

37. N. D. Drasdo and C. W. Fowler, "Non-linear projection of a retinal image in a wide-angle schematic eye," Br. J. Ophthamol. 58, 709-714 (1974).

38. There are no predictions available from the waveguide model for reflectometric $\rho$ as a function of $\lambda$, since Gorrand and Delori ${ }^{14}$ used only $543 \mathrm{~nm}$. Snyder and Pask ${ }^{18}$ studied the variation of $\rho$ as a function of wavelength and showed (for their specific values of cone diameters, $d_{o}=1 \mu \mathrm{m}$ and $d_{i}=3.2 \mu \mathrm{m}$ for the outer and inner segments, respectively, and refractive indices, $n_{i}=1.353, n_{o}=1.430$, and $n_{\text {ipm }}$ $=1.340$ for the inner and outer segment, and the interphotoreceptor matrix, respectively) that $\rho$ increased from wavelengths between $543 \mathrm{~nm}$ and $650 \mathrm{~nm}$. Nevertheless, we have computed that for the same diameters and slightly different indices of refraction (those used by Gorrand and Delori: $n_{i}=1.361, n_{o}=1.419$, and $n_{\mathrm{ipm}}=1.347$ ) the behavior is reversed and $\rho$ decreases with wavelength between 555 and $650 \mathrm{~nm}$, thus indicating that the variation of $\rho$ with $\lambda$ in the waveguide models is non- systematic and strongly dependent on the choice of parameters.

39. A. E. Elsner, S. A. Burns, J. J. Weiter, and F. C. Delori, "Infrared imaging of subretinal structures in the human ocular fundus," Vision Res. 36, 191-205 (1996).

40. The multiplication arises from the fact that at each pupil location the contribution from each retinal location is attenuated by the same waveguide component. That is,

$$
\begin{gathered}
I p(x, y) \mid \int_{\xi} \int_{\zeta} O_{i}(\xi, \zeta) 10-\frac{\rho_{w g}^{2}}{2}\left(x^{2}+y^{2}\right) \\
\quad \times\left.\exp \left[\frac{-i 2 \pi}{\lambda z}(\xi x+\zeta y)\right] \mathrm{d} \xi \mathrm{d} \zeta\right|^{2},
\end{gathered}
$$

where $\operatorname{Ip}(x, y)$ is the intensity distribution at the plane of the pupil, $O_{i}(\xi, \zeta)$ is the complex amplitude of the retinal eccentricity, $(x, y)$ are pupil coordinates, and $(\xi, \zeta)$ are retinal coordinates. Since the waveguide component 10 $-\rho_{w g}^{2}\left(x^{2}+y^{2}\right)$ does not depend on the retinal coordinates, it can be moved out of the integral.

41. C. A. Curcio,"Diameters of presumed cone apertures in human retina," in Annual Meeting, Vol. 20 of 1987 OSA Technical Digest Series (Optical Society of America, Washington, D.C., 1987), p. 83.

42. D. I. MacLeod, "Directionally selective light adaptation: a visual consequence of receptor disarray?" Vision Res. 14, 369-378 (1974).

43. G. Westheimer, "Dependence of the magnitude of the Stiles-Crawford effect on retinal location," J. Physiol. (London) 192, 309-315 (1967).

44. J. M. Enoch and G. M. Hope, "Directional sensitivity of the foveal and parafoveal retina," Invest. Ophthalmol. Visual Sci. 12, 497-503 (1973). 